\title{
Lack of Long-Term Protective Effect of Antioxidant/Anti-Inflammatory Therapy in Transplant-Induced Ischemia/Reperfusion Injury
}

\author{
You-Lin Tain ${ }^{\text {a }}$ Veronika Muller $^{\mathrm{b}}$ Attila Szabo $^{c}$ Anna Dikalova $^{d}$ \\ Kathy Griendling ${ }^{d}$ Chris Baylis $^{a}$ \\ a Department of Physiology and Functional Genomics, University of Florida, Gainesville, Fla., USA; \\ Departments of ${ }^{\mathrm{b}}$ Pulmonology, and ${ }^{\mathrm{C}}$ Pediatrics, Semmelweis University, Budapest, Hungary; and \\ ${ }^{\mathrm{d}}$ Department of Medicine, Division of Cardiology, Emory University School of Medicine, Atlanta, Ga., USA
}

\section{Key Words}

Antioxidant $\cdot$ Ischemia/reperfusion $\cdot$ Kidney transplant •

Nitric oxide

\begin{abstract}
Background: Alloantigen-independent factors contribute to long-term damage in renal transplant recipients, likely due to ischemia/reperfusion (I/R) injury at transplantation (Tx). I/R injury promotes oxidative stress and inflammation resulting in endothelial injury. Methods: In this study we investigated the long-term efficacy (22 weeks) of short-term (10 day) endothelial protection therapy (EP) in 'optimal' donor kidneys using the male Fisher 344 rat isograft (ISO) model. ISO-EP kidneys were compared to untreated ISO (ISO-UN) kidneys. EP involved dexamethasone to donor, ex vivo treatment of the kidney with deferoxamine and tempol, and administration to the recipient of L-arginine and tempol for 10 days. Rats were sacrificed 22 weeks following Tx and compared to age-matched, normal controls. Results: Both groups of ISO Tx rats developed similar renal dysfunction and structural damage and renal NADPH-oxidase-depen-
\end{abstract}

These studies were supported by the National Institutes of Health grants DK56843 and DK 45517 (C.B.), HL38206 (K.G.), and the Hungarian Scientific Research Fund OTKA F042563 (V.M. and A.S.). (c) 2006 S. Karger AG, Basel

$0250-8095 / 06 / 0263-0213 \$ 23.50 / 0$

Fax +41613061234 E-Mail karger@karger.ch www.karger.com
Accessible online at: www.karger.com/ajn dent $\mathrm{O}_{2}^{-}$production was similarly elevated in ISO-UN and ISO-EP groups vs. controls. In vitro renal cortex NO synthase (NOS) activity was also similar in ISO-UN and ISO-EP rats, despite lower nNOS and eNOS protein abundance in ISO-EP. Conclusion: I/R injury-induced late graft dysfunction occurs even when optimal donors are used and when short-term EP treatment is given. Increased renal superoxide production is not prevented by short-term EP therapy.

Copyright $\odot 2006$ S. Karger AG, Basel

\section{Introduction}

Living related donor kidney transplantation (Tx) has better survival than that from cadaver donors, but can still develop chronic allograft nephropathy $[1,2]$. In rats, even isografts in which the donor kidney was harvested under optimal conditions develop structural damage due to ischemia/reperfusion (I/R) injury. Immediately after Tx, post-ischemic reperfusion results in the generation of reactive oxygen and nitrogen species, activation of inflammatory cascades and endothelial injury [3].

Antioxidants and anti-inflammatory agents have been used to prevent I/R [3, 4]. Tempol (4-hydroxy-tempo), a membrane-permeable superoxide dismutase mimetic that removes superoxide $\left(\mathrm{O}_{2}^{-}\right)$and facilitates hydrogen peroxide dismutation at a dose of $30 \mathrm{mg} / \mathrm{kg} / \mathrm{h}$ for $6 \mathrm{~h}$, was 
reported to prevent renal I/R injury [5]. Deferoxamine, an iron chelator, has been included in preservation solutions for $18 \mathrm{~h}$ and reduces renal I/R injury 9 days after Tx [6]. L-Arginine is a substrate for nitric oxide synthase (NOS) and reduces renal I/R injury when administered once before I/R [7]. Glucocorticoids are anti-inflammatory agents and also prevent the induction of the damaging inducible NOS. Although the individual strategies above have been tested to prevent I/R injury and ameliorate short-term graft dysfunction, it is unclear whether these acute effects may affect the long-term graft function. This study investigated whether perioperative (10 day) anti-inflammatory/antioxidant treatment designed to give endothelial protection (EP) would prevent longterm graft injury/dysfunction in renal isograft (ISO) recipients in which kidneys were transplanted under optimal donor conditions.

\section{Materials and Methods}

\section{Animals}

Studies were on 31 inbred male Fisher 344 rats used as donors and recipients for kidney ISO $(\mathrm{n}=24)$ and normal 2-kidney controls $(\mathrm{n}=7)$ studied over the same period $(\mathrm{CON})$. An additional 3 female Fisher and 3 female Lewis rats were used as severe allograft (ALLO) rejection-positive controls for NADPH oxidasedependent $\mathrm{O}_{2}^{-}$determination. All rats were purchased from Harlan Sprague-Dawley (Indianapolis, Ind., USA), and ISO and ALLO were subjected to surgery at 9-14 weeks of age and then followed for 22 weeks. All animals were kept under standard conditions and fed standard rat chow and water ad libitum.

\section{Renal Transplantation}

Rats were anesthetized by intraperitoneal pentobarbital sodium (32.5 mg/kg; Sigma, St. Louis, Mo., USA) and methohexital sodium ( $25 \mathrm{mg} / \mathrm{kg}$; Brevital sodium, Eli Lilly and Co, Indianapolis, Ind., USA). After median laparatomy the left kidney was atraumatically removed. In the donor the abdominal aorta and vena cava were clamped, a cannula placed into the left renal artery and the kidney flushed with cold $\left(4^{\circ} \mathrm{C}\right)$ solution (see below), removed with the artery, vein and ureter and placed into cold solution for $10 \mathrm{~min}$. The donor and recipient renal artery and vein were then anastomized with $10-0$ prolene sutures and after a warm ischemia time of $35 \mathrm{~min}$ (total ischemia time $45 \mathrm{~min}$ ), vascular clamps were removed and the ureter was anastomized end to end near the hilus. To avoid infections, rats received $10 \mathrm{mg} / \mathrm{kg} /$ day rocephin (Ceftriaxone sodium, Roche, Nutley, N.J., USA) for 10 days, then rats were again anesthetized and the right native kidney removed.

\section{Experimental Protocols}

ISO rats were separated into 2 groups: the untreated group (ISO-UN, $\mathrm{n}=6$ ) in which lactated Ringers was used to flush the donor kidney at the time of Tx, and the treated group (ISO-EP, $\mathrm{n}=6$ ) which was given treatments to provide EP. The donors received $10 \mathrm{mg} / \mathrm{kg}$ dexamethasone i.v. (APP, Los Angeles, Calif., USA) 30 min before kidney removal. The donor kidney was flushed with $2 \mathrm{ml} / \mathrm{g}$ kidney weight of cold lactated Ringer's solution containing $1 \mathrm{mM}$ deferoxamine mesylate (Sigma-Aldrich, St. Louis, Mo., USA) and $3 \mathrm{~mm}$ 4-hydroxy-tempo (Sigma-Aldrich), removed and placed in the same cooled solution for $10 \mathrm{~min}$. Recipients were treated with $1 \% \mathrm{~L}$-arginine (Fresenius Kabi Clayton, Clayton, N.C., USA) and $1 \mathrm{~mm} 4$-hydroxy-tempo in the drinking water 1 day before Tx and for 10 days thereafter to provide overall EP. Seven normal male rats were studied over a 22 -week period as CON.

We used additional tissue solely as a positive control for the NADPH oxidase-dependent superoxide production assay. Lewis recipients received renal grafts from Fisher donors (30 min ischemia time) and were treated with cyclosporine (Novartis, Basel, Switzerland) at $1.5 \mathrm{mg} / \mathrm{kg} /$ day for 10 days. The contralateral donor Fisher kidney was harvested at Tx and the allograft was harvested at week 7 because of severe rejection; these provided normal control and severely inflamed samples, respectively.

In all ISO rats, 24-hour urine was collected in metabolic cages for measurement of protein and total $\mathrm{NO}$ production (from $\left.\mathrm{NOx}=\mathrm{NO}_{3}+\mathrm{NO}_{2}\right)$ in the baseline state. All rats were placed on a low NOx diet (AIN 76C, ICN Pharmaceuticals, Inc., Costa Mesa, Calif., USA) for $48 \mathrm{~h}$ before and during the metabolic cage measurements made at weeks 4, 8, 12, 16, 18, 20 and 22 after Tx. CON were studied at baseline and week 22 . Rats were sacrificed under barbiturate anesthesia, an aortic blood sample was collected for measurement of plasma creatinine, blood urea nitrogen, and NOx, and the kidney removed. A section was placed in $10 \%$ buffered formalin for pathology and the remainder separated into cortex and medulla, frozen in liquid nitrogen and stored at $-80^{\circ} \mathrm{C}$ for later analysis.

\section{Analyses}

Creatinine was measured by HPLC as previously described [8], BUN was measured by Sigma Kit 640-A (Sigma Diagnostics) and urine protein by the Bradford assay. Plasma and urine NOx levels were measured by the Greiss reaction.

In vitro NOS activity was measured from conversion of $\mathrm{L}-\left[{ }^{3} \mathrm{H}\right]$-arginine to $\mathrm{L}-\left[{ }^{3} \mathrm{H}\right]$-citrulline in the kidney cortex as described previously [9]. Both supernatant (soluble) and membrane fractions were assayed; the soluble fraction contains predominantly neuronal NOS (nNOS) and inducible NOS, whereas the membrane fraction contains mostly endothelial NOS (eNOS). Samples were run at baseline and in the presence of nonselective NOS inhibitors: $2 \mathrm{mM}$ trifluoperazine, $5 \mathrm{mM} \mathrm{N}^{\mathrm{G}}$-methyl-L-arginine (L-NMA), and $10 \mathrm{mM} \mathrm{N} \mathrm{N}^{\mathrm{G}}$-nitro-L-arginine methyl ester (L-NAME, Sigma-Aldrich). Data are expressed as picomoles citrulline per minute per milligram protein minus any activity not inhibited by the NOS inhibitor cocktail and adjusted for background.

Detection of NOS abundance by Western blot was as follows: nNOS with a rabbit polyclonal antibody [9] (1:10,000 dilution, 1-hour incubation) and a secondary goat anti-rabbit IgG-HRP antibody (Bio-Rad; 1:3,000 dilution, 1-hour incubation). Membranes were stripped and re-probed for eNOS with a mouse monoclonal antibody (Transduction Labs, 1:250 dilution, 1-hour incubation) and a secondary goat anti-mouse IgG-HRP antibody (Transduction Labs, 1:2,000 dilution, 1-hour incubation). Bands of interest were visualized using an enhanced chemilumines-
Tain/Muller/Szabo/Dikalova/ Griendling/Baylis 
Fig. 1. Renal pathology 22 weeks following kidney transplantation. Degree of glomerulosclerosis (\%; A) and Banff score (0-12; B). ${ }^{*} \mathrm{p}<0.05$ vs. CON.

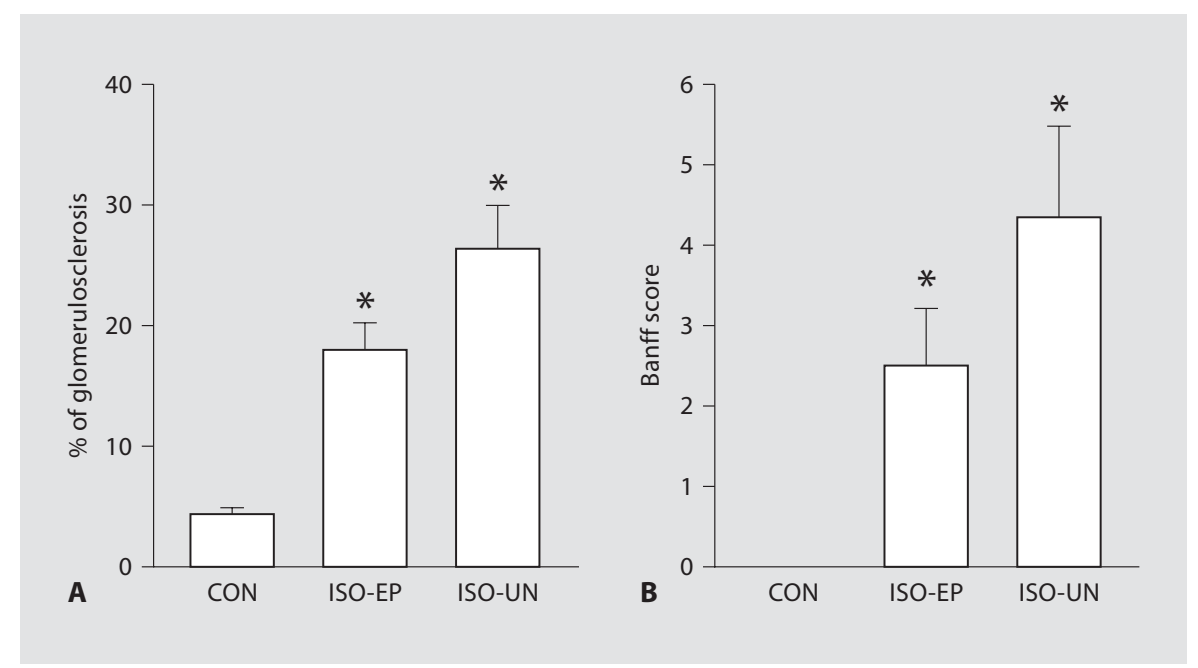

cence reagent and quantified by densitometry, as the integrated optical density (IOD) after subtraction of background. The IOD was factored for Ponceau red staining to correct for any variations in total protein loading and for an internal standard (either cerebellar or endothelial cell lysate). Additional details have been published previously [9].

$\mathrm{NADPH}$-dependent $\mathrm{O}_{2}^{-}$production was measured by electron spin resonance (ESR) spectroscopy. Membrane samples from kidneys were prepared as described previously [10] using the hydroxylamine spin probe $\mathrm{CPH}$ to provide quantitative measurements of $\mathrm{O}_{2}^{-}$radicals with high sensitivity $[10,11]$. The ESR spectra were recorded using an EMX ESR spectrometer (Bruker) and a superhigh-Q microwave cavity.

Pathology was performed on 5- $\mu \mathrm{m}$ sections of formalin-fixed kidney, blocked in paraffin wax, stained with PAS and HE stains as described previously [9]. The level of renal injury was assessed on a blinded basis by calculating sclerotic damage to glomeruli ( $\mathrm{n}=100)$ using the 0 to $4+$ scale and the Banff score which also considers the degree of tubular atrophy, interstitial damage and intimal hyperplasia.

\section{Statistical Analysis}

Results are presented as mean \pm SE. Parametric data was analyzed by unpaired $t$ test. Histological findings were analyzed by Mann-Whitney $U$ test. $\mathrm{p}<0.05$ was considered statistically significant.

\section{Results}

As shown in table 1, ISO-UN and ISO-EP Fisher rats had similar body weights (BW), kidney weights (KW) and $\mathrm{KW} / \mathrm{BW}$ ratio, which was higher than in normal 2 kidney rats due to compensatory hypertrophy. Creatinine clearance, plasma creatinine and BUN were similar in both groups of ISO and lower vs. CON (table 1).
Table 1. Weight and renal function measures 22 weeks following kidney transplantation or similar time in controls

\begin{tabular}{lccc}
\hline & ISO-UN & ISO-EP & CON \\
\hline Body weight, g & $408 \pm 15^{*}$ & $396 \pm 13^{*}$ & $456 \pm 8$ \\
Kidney weight, $g$ & $2.20 \pm 0.14$ & $2.36 \pm 0.16$ & $1.84 \pm 0.14$ \\
Kidney weight/body weight & & & \\
$\quad \times 1,000$ & $5.36 \pm 0.02^{*}$ & $5.96 \pm 0.36^{*}$ & $4.07 \pm 0.35$ \\
Blood urea nitrogen, mg/dl & $23 \pm 1^{*}$ & $21 \pm 1$ & $18 \pm 1$ \\
Plasma creatinine, mg/dl & $0.50 \pm 0.02^{*}$ & $0.44 \pm 0.02^{*}$ & $0.30 \pm 0.02$ \\
CCr/BW, ml/min/kg BW & $4.3 \pm 0.4^{*}$ & $4.6 \pm 0.1^{*}$ & $7.3 \pm 0.5$ \\
UprotV, mg/24 h & $50.7 \pm 9.4$ & $30.7 \pm 4.2^{*}$ & $35.8 \pm 3.9$ \\
\hline
\end{tabular}

Values are represented as mean $\pm \mathrm{SE}$. BW = Body weight; ISO$\mathrm{UN}=$ isograft without therapy; ISO-EP = isograft with endothelial protection; $\mathrm{CON}=$ time control; $\mathrm{CCr}=24$-hour clearance of creatinine; UproV $=24$-hour urine protein.

${ }^{*} \mathrm{p}<0.05$ vs. CON

\# $\mathrm{p}<0.05$ vs. ISO-UN.

Mild proteinuria developed in the ISO-UN rats (table 1) while the ISO-EP group showed no change from baseline or from CON at week 22. By histology, the ISOEP and ISO-UN rats developed moderate and similar glomerulosclerosis and tubulointerstitial injury vs. CON (fig. 1). Despite the lack of proteinuria the EP group was not protected from structural damage compared to the UN rats.

The EP protocol should provide protection against inflammation and oxidative stress before and for the 10 days after $\mathrm{Tx}$, the period where I/R injury would likely be developing. However, as shown in figure 2, by 22 
weeks after Tx there was no difference in renal NADPHdependent $\mathrm{O}_{2}^{-}$production in ISO-EP and ISO-UN kidney cortex. This assay is able to detect oxidative stress since we observed increased renal NADPH-dependent $\mathrm{O}_{2}^{-}$production in female Lewis recipients undergoing severe graft rejection compared to normal kidney CON.

By Western blot, ISO-EP rats showed lower nNOS and eNOS protein abundance in renal cortex vs. ISO-UN (fig. 3A) but the in vitro NOS activity in both soluble (main location of the nNOS) and membrane (main location of the eNOS) fractions were similar between the

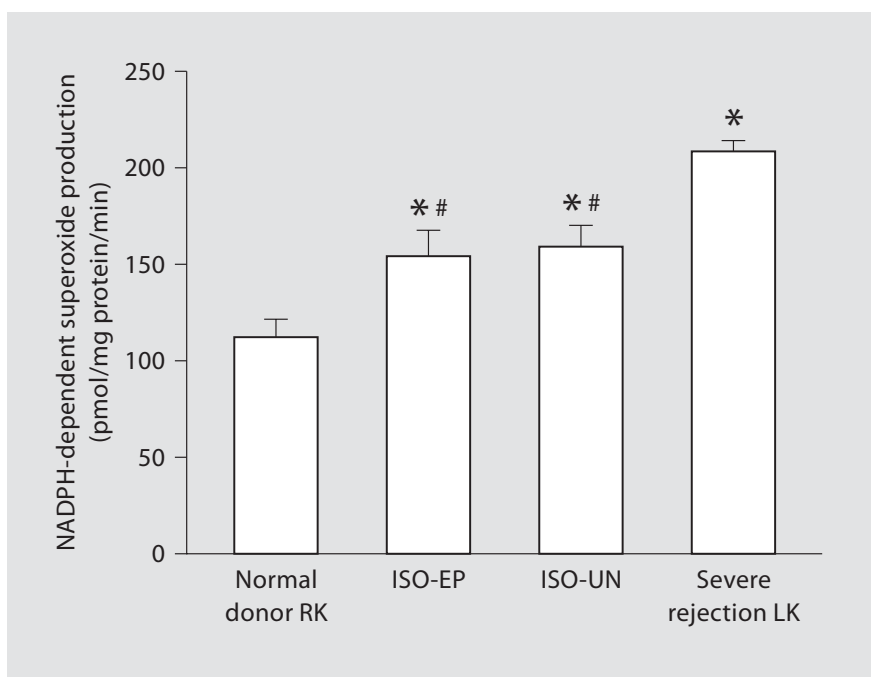

Fig. 2. NADPH-dependent superoxide production of ISO-UN, ISO-EP, and acutely rejecting allografts and the control kidneys. ${ }^{*} \mathrm{p}<0.05$ vs. normal donor $\mathrm{RK} ;{ }^{\#} \mathrm{p}<0.05$ vs. severe rejection LK.
2 groups (fig. 3B). There were no differences between ISO-UN and ISO-EP in the abundance of nNOS and eNOS in the medulla (data not shown).

\section{Discussion}

The main finding in this study is that short-term (10-day) intensive EP with combined antioxidant and anti-inflammatory treatment at the time of Tx has no long-term benefit in terms of structure or function of the Tx-ISO Fisher rat kidney.

While much of the chronic renal Tx damage is antigen-dependent, non-immunological mechanisms also contribute [3]. The condition of the donor and donor kidney are of prime importance and in this study we optimized Tx by taking kidneys from healthy living donors and subjecting them to the minimum necessary cold and warm ischemia time, similar to the procedure used in man. Despite this optimization significant structural damage develops after Tx, even in the absence of immunological mismatch or cytotoxic immunosuppressants since ISO-UN have substantial damage compared to CON after 22 weeks (fig. 1). Because uninephrectomized Fisher kidneys are functionally and morphologically identical to 2-kidney controls [12], the injury must come from Tx-induced I/R injury. Not all strains show susceptibility to I/R injury since optimal ISO kidneys in Brown Norway rats showed normal function and structure 1 year after Tx [13].

Our main goal was to determine whether 10 days of EP during the perioperative period would improve longterm structure/function of ISO kidneys from 'optimal'
Fig. 3. A Relative abundance of renal cortex neuronal (nNOS) and endothelial nitric oxide synthase (eNOS) of ISO-UN and ISO-EP rats. IOD = Integrated optical density; Int Std = internal standard; Ponc = Ponceau red stain. B In vitro NOS activity in the soluble (Sol) and membrane fraction (Mem) of cortical homogenates from ISOUN and ISO-EP rats. ${ }^{*} \mathrm{p}<0.05$ vs. ISOUN.

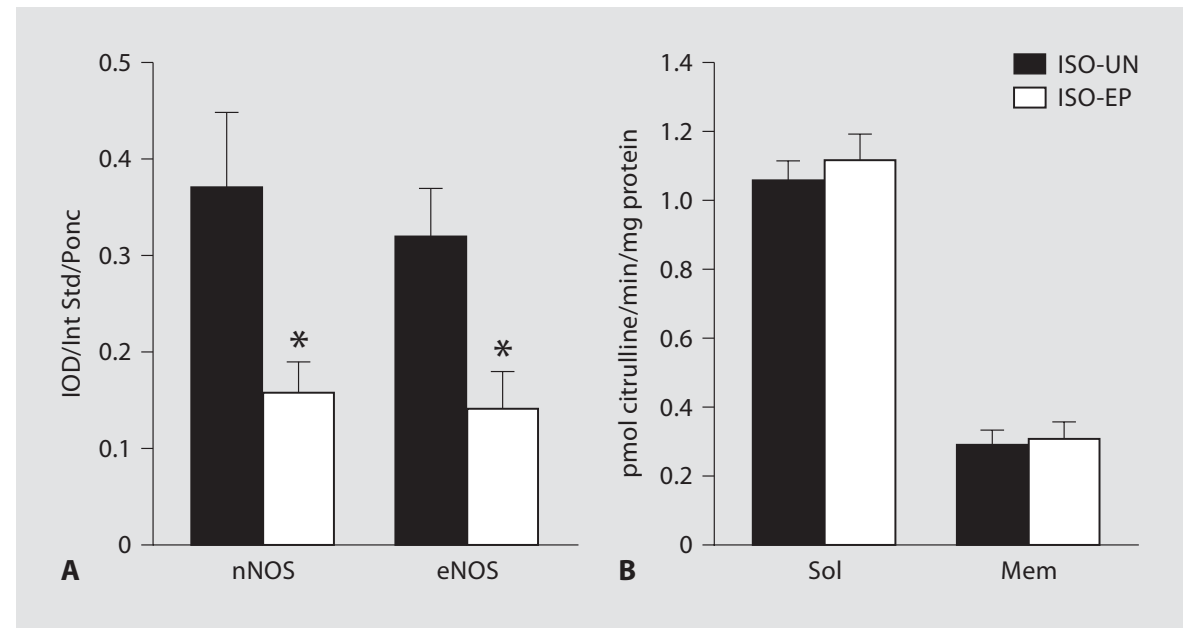


donors. We found that there was no structure/function protection with EP therapy nor was the long-term increase in renal NADPH-dependent $\mathrm{O}_{2}^{-}$production prevented. This contrasts to studies in ALLO (Fisher to Lewis) where function/structure is improved by short-term immunosuppressive/anti-inflammatory treatment of the donor [14]. Although individual agents used in this study have been shown to give short-term renoprotection (hours to 9 days) against I/R injury, their long-term effects are unclear. Short term L-arginine has functional benefit to human ALLO recipients [15]. Thus, our findings suggest that the 'optimal' donor kidney, in the absence of alloreactivity, does not benefit from EP therapy. There is likely to be benefit from treatment of ALLO in man, particularly where 'expanded donor criteria' are used. Moreover, in the present study, although there was no statistically significant improvement in structural damage or functional deterioration, there is a trend to better histology and renal function in the EP group. These findings suggest that longer time follow-up ( $>22$ weeks) or higher numbers of rats may show a small renoprotective effect of EP.

Despite similar structural damage there was a lower renal cortex nNOS and eNOS protein abundance in the treated ISO-EP vs. ISO-UN kidneys. Of note, however, the in vitro NOS activity of both soluble and membrane fractions of renal cortex were similar in ISO-EP and ISOUN. This highlights the complexity of the regulation of NOS enzyme activity which is influenced by many posttranslational modifications as well as by protein abundance. Although the oxidative state is a major regulator of NOS protein abundance and activity, there was no difference in the amount of $\mathrm{O}_{2}^{-}$generated by NADPH oxidase between untreated and treated groups. These findings suggest that EP therapy partially restores NO synthesis and endothelial function so that lower abundance of nNOS and eNOS are required in ISO-EP rat.

In conclusion, short-term EP therapy did not prevent the moderate chronic injury, oxidative stress and falls in function seen when optimal kidneys are grafted into syngeneic recipients.

\section{Acknowledgments}

Technical assistance of Kevin Engels, Gary Freshour, and Lennie Samsell is gratefully acknowledged.

\section{References}

1 Hariharan S, Johnson CP, Bresnahan BA, Taranto SE, McIntosh MJ, Stablein D: Improved graft survival after renal transplantation in the United States, 1988 to $1996 . \mathrm{N}$ Engl J Med 2000;342:605-612.

-2 Halloran PF, Homik J, Goes N, Lui SL, Urmson J, Ramassar V, Cockfield SM: The 'injury response': a concept linking nonspecific injury, acute rejection, and long-term transplant outcomes. Transplant Proc 1997;29: 79-81.

-3 Perico N, Cattaneo D, Sayegh MH, Remuzzi G: Delayed graft function in kidney transplantation. Lancet 2004;364:1814-1827.

-4 Cuzzocrea S, Riley DP, Caputi AP, Salvemini D: Antioxidant therapy: a new pharmacological approach in shock, inflammation, and ischemia/reperfusion injury. Pharmacol Rev 2001;53:135-159.

$\checkmark 5$ Chatterjee PK, Cuzzocrea S, Brown PA, Zacharowski K, Stewart KN, Mota-Filipe H, Thiemermann C: Tempol, a membrane-permeable radical scavenger, reduces oxidant stress-mediated renal dysfunction and injury in the rat. Kidney Int 2000;58:658-673.
-6 Huang H, He Z, Roberts LJ 2nd, Salahudeen AK: Deferoxamine reduces cold-ischemic renal injury in a syngeneic kidney transplant model. Am J Transplant 2003;3:1531-1537.

7 Chander V, Chopra K: Renal protective effect of molsidomine and L-arginine in ischemia-reperfusion induced injury in rats. J Surg Res 2005;128:132-139.

8 Erdely A, Wagner L, Muller V, Szabo A, Baylis C: Protection of Wistar Furth rats from chronic renal disease is associated with maintained renal nitric oxide synthase. J Am Soc Nephrol 2003;14:2526-2533.

-9 Szabo AJ, Wagner L, Erdely A, Lau K, Baylis $\mathrm{C}$ : Renal neuronal nitric oxide synthase protein expression as a marker of renal injury. Kidney Int 2003;64:1765-1771.

10 Hanna IR, Hilenski LL, Dikalova A, Taniyama Y, Dikalov S, Lyle A, Quinn MT, Lassegue B, Griendling KK: Functional association of nox1 with p22phox in vascular smooth muscle cells. Free Radic Biol Med 2004;37:1542-1549.

11 Dikalov SI, Dikalova AE, Mason RP: Noninvasive diagnostic tool for inflammation-induced oxidative stress using electron spin resonance spectroscopy and an extracellular cyclic hydroxylamine. Arch Biochem Biophys 2002;402:218-226.
12 Azuma H, Nadeau K, Takada M, Mackenzie HS, Tilney NL: Cellular and molecular predictors of chronic renal dysfunction after initial ischemia/reperfusion injury of a single kidney. Transplantation 1997;64:190197.

13 Kouwenhoven EA, de Bruin RW, Heemann U, Marquet RL, Ijzermans JN: Does cold ischemia induce chronic kidney transplant dysfunction? Transplant Proc 1999;31:988989.

14 Reutzel-Selke A, Zschockelt T, Denecke C, Bachmann U, Jurisch A, Pratschke J, Schmidbauer G, Volk HD, Neuhaus P, Tullius SG: Short-term immunosuppressive treatment of the donor ameliorates consequences of ischemia/reperfusion injury and long-term graft function in renal allografts from older donors. Transplantation 2003;75:17861792.

15 Schramm L, La M, Heidbreder E, Hecker M, Beckman JS, Lopau K, Zimmermann J, Rendl J, Reiners C, Winderl S, Wanner C, Schmidt HH: L-Arginine deficiency and supplementation in experimental acute renal failure and in human kidney transplantation. Kidney Int 2002;61:1423-1432. 